\title{
Sistema de monitoreo remoto de sensores de temperatura y corriente usando una red híbrida bus CAN-Zigbee
}

\author{
Héctor Anzures Martínez, Ángel Bulmaro Sánchez Rubio, Josefina Castañeda \\ Camacho, Gerardo Mino Aguilar, José Fermi Guerrero Castellanos, Liliana \\ Cortez
}

\author{
Benemérita Universidad Autónoma de Puebla, \\ Facultad de Ciencias de la Electrónica, Puebla, \\ México
}

\begin{abstract}
Resumen. Este trabajo trata sobre un sistema de monitoreo remoto a través de una pantalla inteligente para sensores de temperatura y corriente utilizando una red híbrida CAN-ZIGBEE. El CAN bus es usado como medio de transmisión de datos a corta distancia mientras que Zigbee es empleado para que cada nodo de la red pueda interactuar de manera inalámbrica con el nodo principal. De esta manera la red híbrida combina las ventajas de cada protocolo de comunicación para intercambiar datos. El sistema cuenta con cuatro nodos, dos son CAN y reciben la información de los sensores y el resto son Zigbee. Estos nodos están a cargo de transmitir la información de un nodo CAN de manera inalámbrica y desplegarla en una pantalla inteligente.
\end{abstract}

Palabras clave: CAN bus, Zigbee, dsPIC, monitoreo, pantalla inteligente.

\section{System for Remote Monitoring of Temprature and Current Sensors Using Hybrid Red Bus CAN- Zigbee}

\begin{abstract}
This paper describes a remote monitoring system that uses an intelligent screen with temperature and current sensors and a CAN bus-Zigbee hybrid network. CAN bus is used as short distance data transmission medium, while Zigbee is used in a way that every node in the network can interact wirelessly with the main node. In this way, the network combines the advantages of every single communication protocol for data interchange. The system is composed by 4 nodes, 2 nodes are CAN and they receive the sensors' information and the rest of the nodes are Zigbee. These nodes are responsibles of transmitting information of a CAN node wirelessly and display it on an intelligent screen.
\end{abstract}


Keywords: CAN bus, Zigbee, dsPIC, monitoring, intelligent screen.

\section{Introducción}

En la actualidad el uso de sistemas de monitoreo para distintas aplicaciones es importante debido a que proporcionan al usuario una interfaz para poder visualizar el funcionamiento de dicho sistema y detectar posibles errores. Un punto esencial para estos sistemas es la obtención de los resultados de manera sencilla. El bus CAN es un protocolo de comunicación serial con topología de bus diseñado para el intercambio de información entre múltiples unidades de control electrónico dentro de un sistema distribuido, las principales ventajas de este protocolo son: robustez ante interferencias electromagnéticas, intercambio de información en tiempo real, transmisión de hasta 8 bytes por trama, estandarizado, topología bus que reduce considerablemente el cableado, etc. Las características que presenta el CAN Bus lo han ubicado como protocolo de facto en la industria automotríz y la industria de la automatización [1].

La tecnología Zigbee fué creada por la Zigbee Alliance en el 2004 [2]. Esta tecnología representa una opción atractiva dentro de las redes WPAN (Red de Área Personal) [3,4]. Zigbee se basa en el protocolo IEEE 802.15.4 y es un tópico importante en la investigación de tecnologías de comunicación inalámbrica de corto alcance. Sus principales ventajas son el bajo consumo de potencia, auto organización de baja complejidad y su bajo costo. Es ampliamente utilizada en la automatización de edificios, monitoreo y control de hospitales y hogares, a corta distancia. En este trabajo se describe el diseño e implementación de un sistema de monitoreo de sensores de corriente y temperatura a través de una red híbrida bus CAN-Zigbee.

\section{Diseño del sistema}

\subsection{Diagrama a bloques del sistema}

La Figura 1 muestra el diagrama a bloques del sistema de monitoreo integrado por 4 nodos. Cada nodo está formado por un dsPIC30F3011 y un transceptor CAN MCP2551. El nodo 1 lee la información del sensor 1 (sensor de corriente). Mientras que el nodo 2 lo hace de un sensor de temperatura. Ambos nodos envían información hacia el nodo central. De aquí se envía a un transmisor Zigbee que lo transmite inalámbricamente hacia su correspondiente receptor Zigbee. Éste dispositivo está conectado vía serial con una pantalla LCD donde se despliega la información de los sensores de la red.

\section{2. $\quad$ Red CAN}

Los nodos de la red CAN [5] estón integrados por el dsPIC30F4013 el cual contiene un módulo CAN en su arquitectura para poder establecer comunicación 


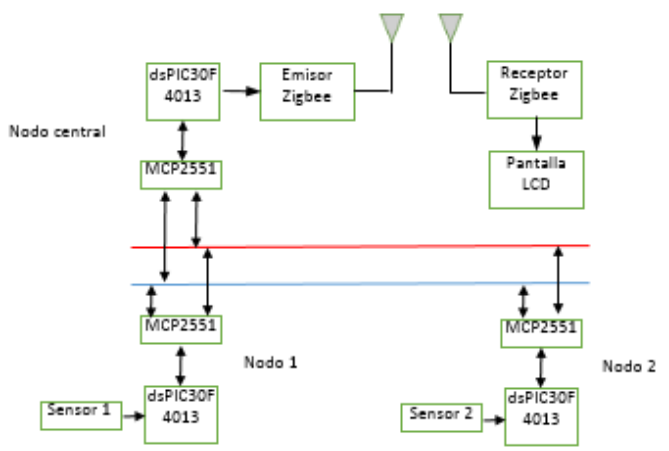

Fig. 1. Diagrama a bloques del sistema.

con otros dispositivos y el transceptor MCP2551 encargado de convertir los niveles de tensión entregados por el dsPIC a los niveles de tensión que necesita una red CAN bus. La Figura 2 muestra la topologá utilizada.

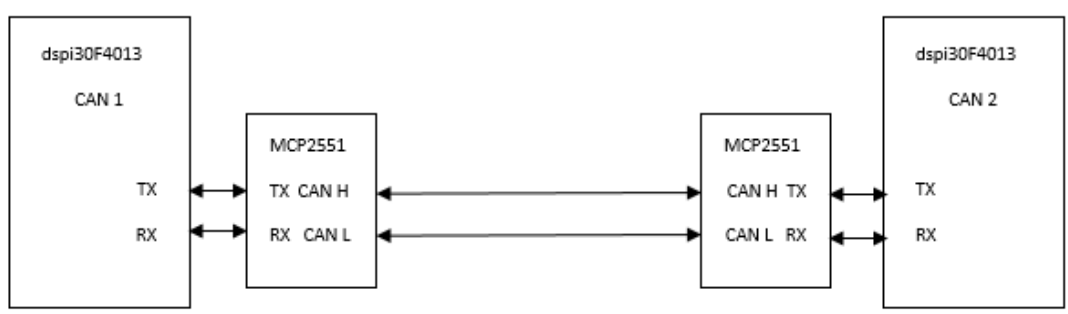

Fig. 2. Topología de red CAN.

\subsection{Matríz CAN}

Para la red CAN se diseñó la matríz de comunicación, en esta matríz se especifica los mensajes que se transmitirán a través de la red, ID, DLC y señales que transportarán las tramas. La Tabla 1 muestra la información de esta matríz.

\subsection{Red Zigbee}

Los módulos Zigbee utilizados, han sido diseñados para cumplir con la norma IEEE 802.15.4. Lo que significa bajo costo, bajo consumo de energía y seguridad. Este dispositivo posee 20 pines y opera dentro de la banda de frecuencia ISM de $2.4 \mathrm{GHz}$. Tiene la capacidad de transmisión de datos 100 metros con una tasa de 250.000 bps. Los módulos Zigbee operan en cinco modos. Cuando no 
Héctor Anzures Martínez, Ángel Bulmaro Sánchez Rubio, Josefina Castañeda Camacho, et al.

Tabla 1. Matríz de comunicación CAN.

\begin{tabular}{|c|c|c|c|c|c|c|c|c|c|}
\hline Id & Tx & $\mathrm{Rx}$ & Dlc & Señal & $\begin{array}{l}\text { Bit de } \\
\text { inicio }\end{array}$ & $\begin{array}{l}\text { Bits de } \\
\text { la señal }\end{array}$ & $\begin{array}{c}\text { Valor } \\
\text { minimo }\end{array}$ & $\begin{array}{c}\text { Valor } \\
\text { máximo }\end{array}$ & Interpretación \\
\hline 069 & CAN1 & $\begin{array}{l}\text { Nodo } \\
\text { central }\end{array}$ & 4 & $\begin{array}{l}\text { Cemperatura } \\
\text { y corriente }\end{array}$ & 0 & 15 & 0 & 255 & Valor digital \\
\hline 073 & CAN2 & $\begin{array}{l}\text { Nodo } \\
\text { central }\end{array}$ & $4^{\mathrm{T}}$ & $\begin{array}{l}\text { Cemperatura } \\
\text { y corriente }\end{array}$ & 0 & 15 & 0 & 255 & Valor digital \\
\hline
\end{tabular}

reciben o transmiten datos, el módulo de RF está en modo inactivo. El módulo de RF cambia a los otros modos de operación bajo diversas condiciones. En el modo de transmisión de datos en serie los datos son recibidos en el pin DI (Data In) y se almacenan en el buffer DI hasta que puedan ser procesados. Cuando el buffer DI llega 17 bytes, de forma predeterminada, el pin CTS pasa a estado lógico alto para indicar al dispositivo host que detenga el envío de datos. En modo recepción, los datos se reciben a través de la antena. Cuando un dato RF es recibido entra por DO (Data Out). La red Zigbee que se utiliza consta de 2 dispositivos que establecen una comunicación punto a punto. Se hace uso de módulos XBEE serie 2 [6] para implementar esta red. La Figura 3 muestra la red Zigbee que se utiliza.

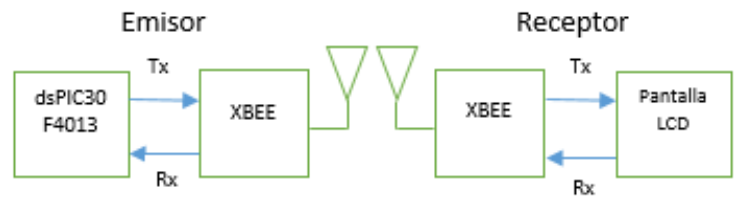

Fig. 3. Diagrama de bloques de la red Zigbee.

\subsection{Interfaz de usuario}

La Interfaz de Usuario consiste en la forma en que el usuario interactúa con el sistema de monitoreo de sensores. La forma más simple es haciendo uso de una pantalla táctil en la que se muestren la información que recolectan los sensores. El diseño de la Interfaz de Usuario se ajusta a una pantalla específica que en este caso corresponde al modelo uLCD-70DT del fabricante 4D Systems.

Se trata de una pantalla de 7 pulgadas con las siguientes características:

- Procesador gráfico DIABLO16.

- Puerto de entradas y salidas de propósito general

- Niveles de voltaje TTL.

Para diseñar la interfaz se requiere el uso del software Workshop creado por el mismo fabricante. Este software posee 4 modos de programación de sus pantallas. 
Sistema de monitoreo remoto de sensores de temperatura y corriente usando una red hibrida bus ...

En este trabajo se utiliza el modo Visi-Genie ya que la idea de la interfaz es que sea lo más simple y atractiva para que el usuario pueda interactuar rápidamente con ella. Este modo de programación nos brinda un estilo gráfico a base de botones, switches, indicadores, etc.

El flujo del diseño de la Interfaz de Usuario obedece a la Fig. 4 . Al inicio se selecciona el modelo de pantalla para que el software ajuste la ventana de diseño al tamaño real de la pantalla, luego se escoge el modo de programación de ésta, en nuestro caso es Visi-Genie. Continuamos con la configuración de los pines del puerto GPIO como entradas o como salidas según se requiera. Luego se agregan los botones e indicadores que servirán para mostrar el estado de los sensores de temperatura y corriente. Posteriormente se asocia cada botón e indicador a un pin del puerto GPIO para que responda a cada evento que el usuario ordene. Finalmente, el puerto serial lee señales digitales analógicas provenientes de los sensores.

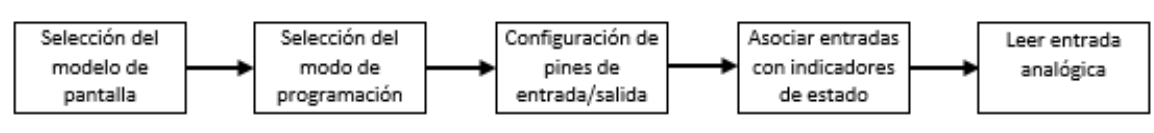

Fig. 4. Flujo de diseño.

\subsection{Sensor de corriente}

El sensor de corriente que se utiliza es el modelo ACS712ELC-30A del fabricante Allegro. Este sensor realiza el sensado de corriente mediante efecto hall [7]. En la Figura 5 se muestra el esquema de conexión de este sensor.

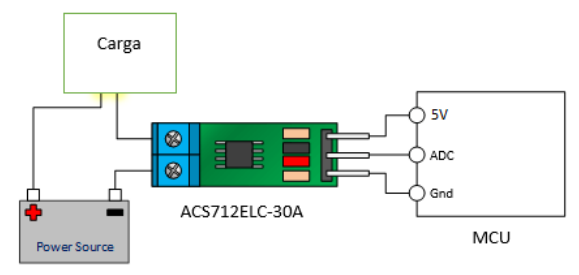

Fig. 5. Esquema de conexión del sensor ACS712ELC-30A.

Este sensor entrega en el pin de salida un voltaje proporcional a la corriente que mide. En la gráfica de la Figura 6 podemos observar la relación entre el voltaje de salida y la corriente sensada. 


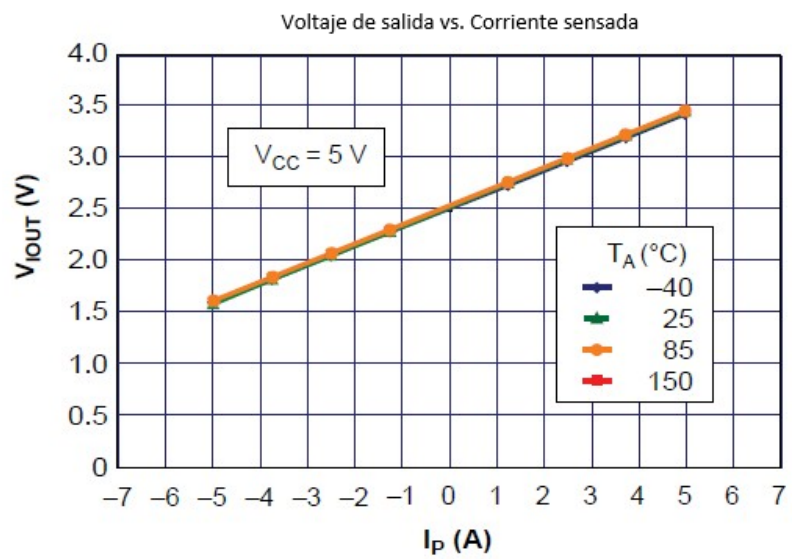

Fig. 6. Relación entre en voltaje de salida y la corriente sensada.

\subsection{Sensor de temperatura}

El LM35 es un sensor de temperatura con una precisión calibrada de $1^{\circ} \mathrm{C}$. $\mathrm{Su}$ rango de medición abarca desde $-55^{\circ} \mathrm{C}$ hasta $150^{\circ} \mathrm{C}$. La salida es lineal y cada grado Celsius equivale a $10 \mathrm{mV}$, por lo tanto:

$150{ }^{\circ} \mathrm{C}=1500 \mathrm{mV}$ y $-55^{\circ} \mathrm{C}=-550 \mathrm{mV}$

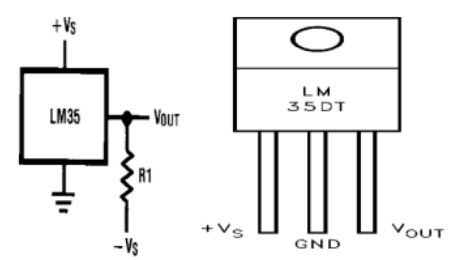

Fig. 7. Simbolo del sensor de temperatura LM35.

\section{Pruebas y resultados}

El sistema de monitoreo de sensores de corriente y temperatura se ha implementado en el hardware que se menciona en las secciones anteriores. Se han realizado pruebas de Indicador de Fuerza de la Señal Recibida(RSSI). Estas pruebas se muestran en las Figs. 8 y 9.

En la Fig. 8 se muestra la prueba RSSI en un ambiente libre de obstáculos. Se transmiten 100 paquetes de datos y durante 1 segundo, que es la duración de esta 


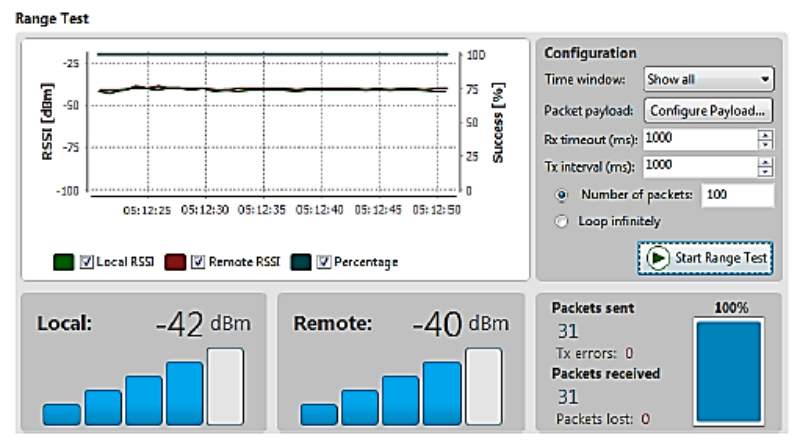

Fig. 8. Prueba de RSSI en ambiente sin obstáculos.

prueba, se reciben 31 y no hay pérdia de datos [8]. La potencia del sistema, de -42 y $-40 \mathrm{dBm}$ para el dispositivo local y remoto respectivamente, se encuentran en el rango de máxima potencia que puede ser recibida en una red inalámbrica.

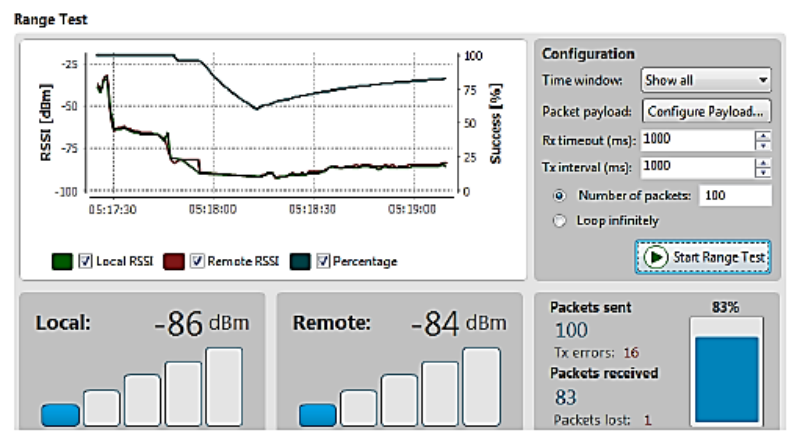

Fig. 9. Prueba de RSSI en ambiente con obstáculos.

La Fig. 9 muestra la prueba RSSI realizada en un ambiente con obstáculos(paredes de concreto, madera, entre otros). En este caso se envía el mismo número de paquetes y se usa el mismo tiempo que en la prueba anterior. El resultado muestra una pérdida de potencia, cayendo a $-86 \mathrm{dBm}$ y $-84 \mathrm{dBm}$. Estos valores se encuentran en el rango mínimo acaptable para establecer una conexión inalámbrica.

Por otro lado, con la ayuda de un osciloscopio se verifica que la transmisión de las tramas CAN coincidan con la matríz de comunicación que se desarrolló. En las Figuras 10 y 11 se muestran algunas de las tramas CAN que se obtuvieron con el osciloscopio, la señal en color amarillo que podemos observar corresponde a la línea $C A N_{L}$ del CAN Bus y su respectiva decodificación aparece dentro de las líneas azules. 
Héctor Anzures Martínez, Ángel Bulmaro Sánchez Rubio, Josefina Castañeda Camacho, et al.

Los datos que nos brinda el osciloscopio por medio de la decodificación son:

- El ID del mensaje que se está transmitiendo.

- El código de longitud de datos (DLC), los bytes de información.

- Un checksum.

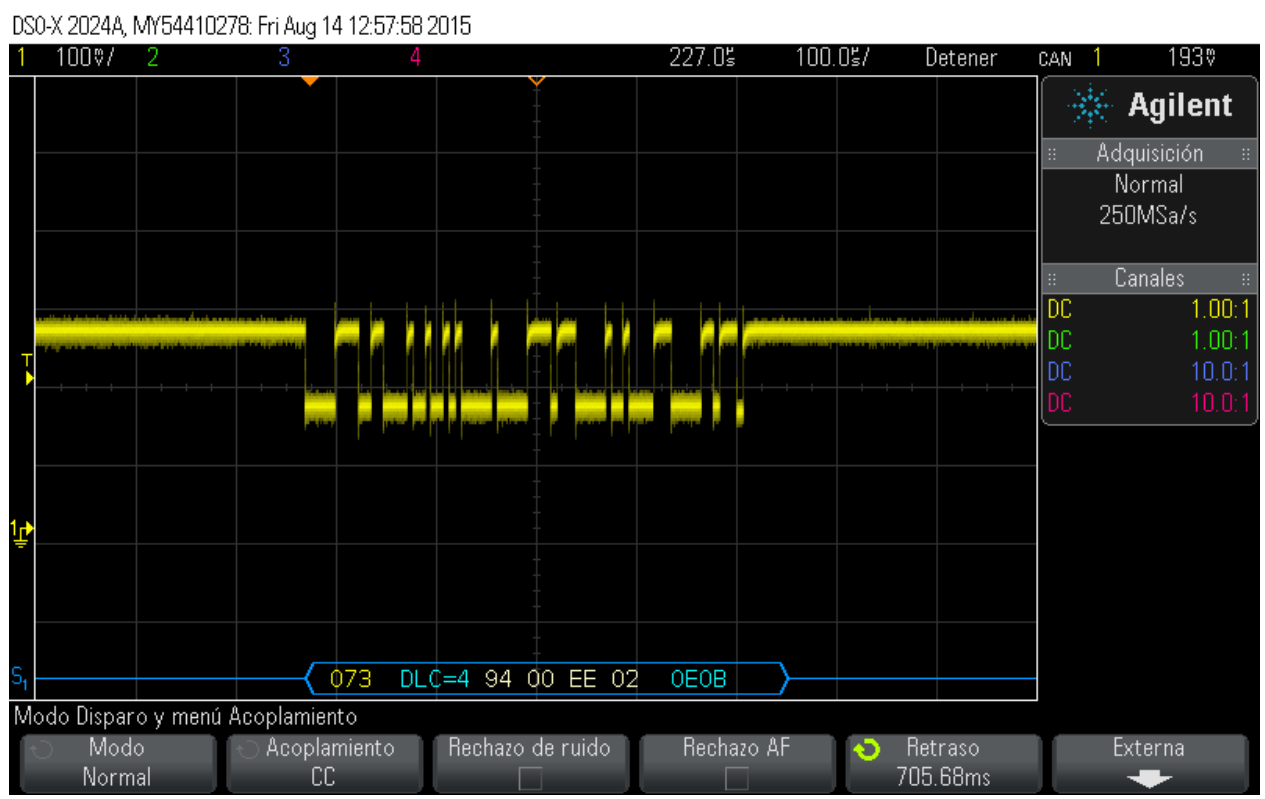

Fig. 10. Trama CAN antes.

En la Fig. 10 podemos ver que el ID del mensaje es 073, un DLC de 4 Bytes que corresponde con la información de dos sensores, la conversión analógico a digital se llevó acabo en 10 bits por lo que se necesitaron 2 bytes por sensor.

En la Fig. 11 podemos observar como a cambiado la información de los sensores pero se conserva el ID del mensaje y el DLC de esta manera comprobamos que la información es correcta.

En la Fig. 12 se muestra la red CAN-Zigbee implementada. La Fig. 12a contiene los tres nodos CAN y el nodo transmisor de Zigbee mientras que la Fig. $12 \mathrm{~b}$ el nodo receptor de Zigbee conectado con la pantalla que posee la interfaz de usuario.

\section{Conclusión}

Durante las pruebas realizadas el sistema mostró el funcionamiento esperado. Las pruebas de RSSI y de transimsión de datos para Zigbee y CAN respectivamente muestran un adecuado desempeño de ambos protocolos de comunicación. 
Sistema de monitoreo remoto de sensores de temperatura y corriente usando una red hibrida bus ...

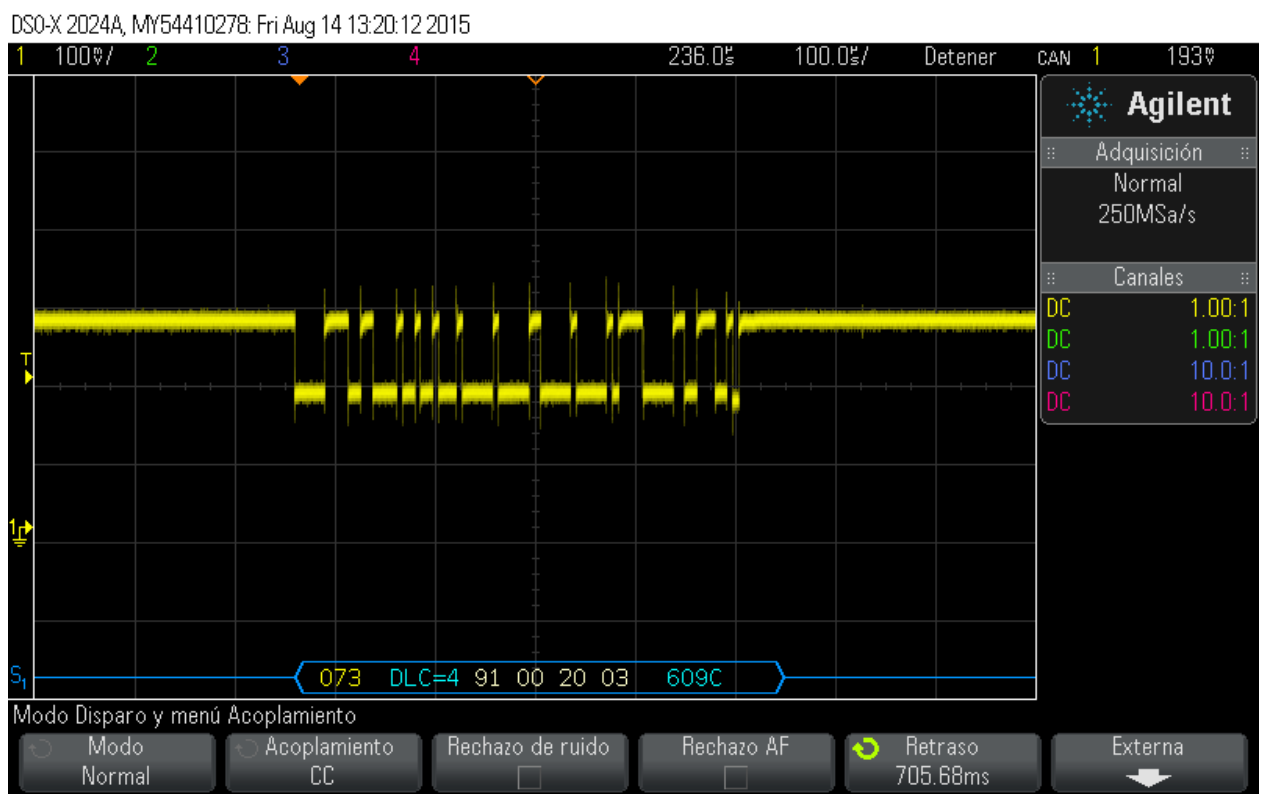

Fig. 11. Trama CAN después.

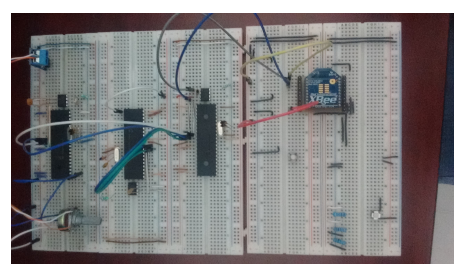

(a) Nodos CAN y Zigbee

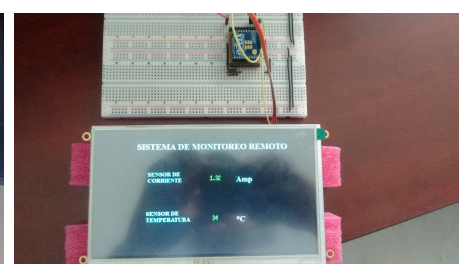

(b) Nodo remoto Zigbee

Fig. 12. Implementación de la red CAN-Zigbee.

En el caso de Zigbee se observó que la calidad del enlace decrece en relación a la distancia entre los nodos Zigbee y los objetos que interpongan en su paso. El bus CAN al tratarse de un protocolo alámbrico no presenta el problema de Zigbee y es por esta otras ventajas que es ampliamente utilizado en sistemas que requiren cierto grado de demanda en la transmisión de datos y confiabilidad. El monitoreo de los sensores de temperatura y corriente se realiza con un muestreo cada 50 ms pantalla, mostrando así la capacidad de la red CAN para transmitir grandes cantidades de información sin pérdidas de datos.

El sistema desarrollado en este trabajo puede ser mejorado agregando más nodos a la red. Al hacer esto se podría realizar muestreo de otras variables provenientes de diversos sensores. También se podrían agregar acciones provenientes de la pantalla de la interfaz de usuario para realizar alguna tarea cuando algún sensor alcance o sobrepase algún valor de umbral preestablecido. La escalabilidad 
Héctor Anzures Martínez, Ángel Bulmaro Sánchez Rubio, Josefina Castañeda Camacho, et al.

de la red es amplia pues Zigbee soporta un numero máximo de 65000 nodos mientras que CAN hasta 30.

\section{Referencias}

1. Robert Bosch GmbH, CAN Specification Version 2.0 (1991)

2. Zigbee technology, Alliance Zigbee. Available at:http://zigbee.org/ (Accessed on June 23, 2015).

3. Li, Jianpo and Zhu, Xuning and Tang, Ning and Sui, Jisheng: Study on ZigBee network architecture and routing algorithm. Signal Processing Systems.

4. Ahamed, SS Riaz: The role of zigbee technology in future data communication system. Journal of theoretical and applied information technology, 129-135 (2010)

5. Tindell, K.W., Hansson, H., Wellings, A.J.: Analysing real-time communications: controller area network (CAN). In: IEEE Real-Time Systems Symposium, pp. 259263 (1994)

6. Oyarce, A., Aguayo, P., Martin, E.: Guía del usuario Xbee series 2. Ingeniería MCI Ltda (2010)

7. Ramón Pallás, A.: Sensores y acondicionadores de señal. Marcombo (2004)

8. Hyncica, O., Kacz, P., Fiedler, P., Bradac, Z., Kucera, P., Vrba, R.: The Zigbee experience. In: Proceedings of the 2nd International Symposium on Communications, Control, and Signal Processing (2006) 